\title{
Many-Body Interactions in the CdTe-Based Quantum Well under Strong Optical Excitation
}

\author{
P. PŁochocka ${ }^{a, *}$, P. Kossacki ${ }^{a}$, B. Piechal ${ }^{a}$, W. Maślana ${ }^{a, b}$,

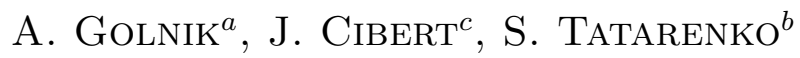 \\ AND J.A. GAJ ${ }^{a}$ \\ ${ }^{a}$ Institute of Experimental Physics, Warsaw University \\ Hoża 69, 00-681 Warsaw, Poland \\ ${ }^{b}$ Laboratoire de Spectrométrie Physique, CNRS \\ et Université Joseph Fourier, Grenoble \\ B.P. 87, 38402 Saint Martin d'Hères Cedex,France \\ ${ }^{c}$ Laboratoire Louis Néel, CNRS, B.P.166, 38042 Grenoble cedex, France
}

\begin{abstract}
We performed pump-probe time-resolved absorption measurements on a modulation $p$-doped $(\mathrm{Cd}, \mathrm{Mn}) \mathrm{Te}$ quantum well. The density of the $2 \mathrm{D}$ hole gas was controlled, in the $10^{10} \mathrm{~cm}^{-2}$ range, by additional $\mathrm{cw}$ illumination. Interactions between photocreated neutral $(X)$ and charged $\left(X^{+}\right)$excitons and the $2 \mathrm{D}$ hole gas were analyzed. We found that the strongest effect is the influence of the carriers on both $X$ and $X^{+}$optical resonances. Neutral and charged excitons are screened by holes, which results in the decrease in their intensities. We conclude from polarization resolved experiments that this screening is spin-dependent: it is more efficient between holes with opposite spins. Binding holes into the charged excitons reduces the screening of the neutral excitons and leads to an enhancement of the neutral exciton intensity. We also analyzed weaker effects, due to exciton-exciton interaction, at a constant hole density. We found that the reduction of the neutral exciton intensity due to two different mechanisms (phase-space filling and biexciton formation) is almost equal. We observed a spin-dependent blue shift of the neutral exciton line in the presence of a population of neutral excitons created by the pump pulse. Due to the attractive interaction between excitons with opposite spins, the corresponding shift of the neutral exciton is smaller than that observed due to the interaction between excitons with the same spins.
\end{abstract}

PACS numbers: 78.67.De, 71.35.Pq, 78.47.+p

*corresponding author; e-mail: Paulina.Plochocka@fuw.edu.pl 


\section{Introduction}

In doped quantum wells (QW's) optical spectra are often dominated by the charged exciton (trion) line. The first experimental identification of charged excitons in a QW was done by Kheng et al. [1]. They observed negative trion lines in photoluminescence and reflectivity spectra of modulation doped $\mathrm{CdTe} /(\mathrm{Cd}, \mathrm{Zn}) \mathrm{Te}$ QWs. The observation of the trion line in optical spectra was also reported in GaAs QWs [2,3]. In further optical studies positive trions were also observed in CdTe-based QWs $[4,5]$. The optical properties of doped QWs were investigated by many different experimental techniques such as transmission or reflectivity [6-8], four wave mixing [9-11], photoluminescence [12-15] or pump-probe experiments $[16,17]$.

Under interband optical excitation, three subsystems coexist in a doped QW: trions, neutral excitons, and free carriers. They interact with each other. In order to investigate these interactions one can modify the carrier density or the occupation of the excitonic states. It was experimentally shown that the carrier density influences the position and intensity of the neutral and charged exciton absorption lines [5]. It was also demonstrated that the intensity of the neutral exciton is reduced in the presence of charged exciton absorption. This effect was called oscillator strength stealing (OSS) and was discussed by many authors $[1,5,8,18,19]$, for both positive and negative trions.

The population of photo-created excitons is usually very small in continuous wave experiments. Therefore, only the influence of preexisting carriers on the neutral and charged exciton can be analyzed under such conditions. A strong optical excitation has to be used in order to analyze the effect of occupation of the excitonic states. In this paper we present results of pump-probe experiments on a $p$-doped $(\mathrm{Cd}, \mathrm{Mn}) \mathrm{Te} \mathrm{QW}$. By using a strong optical excitation, we analyzed the effect of a selective filling of the excitonic states on the optical resonances. This analysis was done for different values of the hole density.

\section{Experiment}

The modulation doped structure studied consists of a single $80 \AA \mathrm{QW}$ of $\mathrm{Cd}_{1-x} \mathrm{Mn}_{x} \mathrm{Te}(x=0.0018)$ embedded between $\mathrm{Cd}_{0.66} \mathrm{Zn}_{0.07} \mathrm{Mg}_{0.27} \mathrm{Te}$ barriers, the whole structure being grown pseudomorphically on a $(100) \mathrm{Cd}_{0.88} \mathrm{Zn}_{0.12} \mathrm{Te}$ substrate. Due to strain and confinement, the energy of light hole excitons is larger than that of heavy hole ones by more than $35 \mathrm{meV}$. Therefore only effects related to heavy holes are important in our experiments. Modulation $p$-type doping was assured by a nitrogen-doped layer placed at $200 \AA$ from the QW. The density of the hole gas in the QW was additionally controlled by illumination with a tungsten halogen lamp, with a blue filter so that the photons have an energy larger than the bandgap of the barriers. Achieved hole densities ranged from $10^{10} \mathrm{~cm}^{-2}$ up to $10^{11} \mathrm{~cm}^{-2}$. The mechanism that allows us to control the carrier density, and 
its calibration, have been described in detail in [5, 14]. The sample was mounted strain-free in a superconducting magnet and immersed in superfluid helium at $1.8 \mathrm{~K}$.

Light pulses were generated by a $\mathrm{Ti}^{3+}: \mathrm{Al}_{2} \mathrm{O}_{3}$ laser operating at $765 \mathrm{~nm}$ $(1620 \mathrm{meV})$, at a repetition rate of $100 \mathrm{MHz}$. The $100 \mathrm{fs}$ laser pulse had a spectral width of about $40 \mathrm{~nm}(85 \mathrm{meV})$. It was spectrally much broader than the splitting between the neutral and charged excitons. The pump and probe pulses were focused on the sample to a common spot of diameter of about $100 \mu \mathrm{m}$. The spectrum of the probe pulse, transmitted through the sample, was recorded as a function of the pump-probe delay. Selective, resonant excitation of the neutral exciton or of the charged exciton was achieved by shaping the laser pulse to a spectral width less than $1 \mathrm{~nm}$ (less than $2 \mathrm{meV}$ ), and a duration of about $2 \mathrm{ps}$. The power of both pulses was controlled independently. After the shaping of the pump pulse, its average power

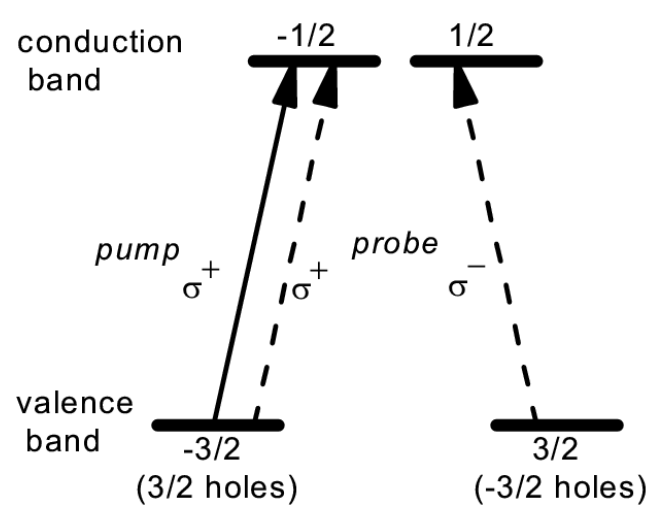

Fig. 1. Optical transitions for the circular polarizations of the pump and probe pulses.

was $300 \mu \mathrm{W}$, corresponding to the creation of a few times $10^{10} \mathrm{~cm}^{-2}$ excitons by each pulse. We estimated that the number of excitons created by the probe pulse was at least 20 times smaller than the number of excitons created by the pump pulse. The pump beam was circularly polarized. The $\sigma^{+}$polarized pump beam creates electrons with spin $-1 / 2$ in the conduction band, and holes of momentum $+3 / 2$, as shown in Fig. 1 . The probe beam was detected behind the sample in both circular polarizations, thus measuring the absorption associated with the creation of $X$ or $X^{+}$, and producing electron-hole pairs of the same spin as those created by the pump pulse, or opposite.

\section{Continuous wave experiments}

We start the description with the results obtained in continuous wave experiments, in which the transmission of white light through the sample was recorded 

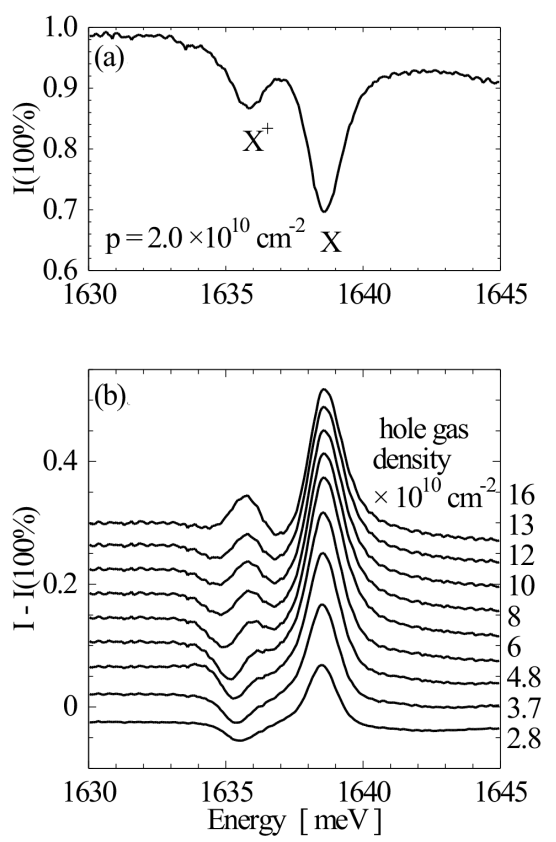

Fig. 2. Typical cw transmission spectrum (a). Difference transmission spectra for different values of the hole density (b).

for different values of the hole density [5]. Figure 2a shows a typical transmission spectrum. The hole density was $2 \times 10^{10} \mathrm{~cm}^{-2}$. Two transitions are observed: the line at higher energy is due to the creation of neutral excitons, the line at lower energy due to the creation of charged excitons. Figure $2 \mathrm{~b}$ shows the difference spectra for different values of the hole density. Such difference spectra were obtained by subtracting the transmission spectrum for the lowest value of the hole density $\left(2 \times 10^{10} \mathrm{~cm}^{-2}\right)$ from the spectra for higher values. Thus we could analyze precisely the relative changes of the position and the intensity of the line. A positive signal in such difference spectra represents a decrease in the line intensity.

It is clear from Fig. $2 \mathrm{~b}$ that the intensity of the neutral exciton line decreases with increasing hole density. Simultaneously the intensity of the charged exciton line initially increases. However, for hole densities higher than $8 \times 10^{10} \mathrm{~cm}^{-2}$, the charged exciton line starts to decrease and eventually disappears: This is due to the filling of optically active states in the $\boldsymbol{k}$ - vector space [15]. In the hole density range, where both the charged and the neutral exciton lines are present, $\left(p \leq 4 \times 10^{10} \mathrm{~cm}^{-2}\right)$, we observe that the decrease in the intensity of $X$ is accompanied by the increase in the intensity of $X^{+}$: This is the oscillator strength stealing. The increase in the charged exciton intensity with carrier density is easily understood: It results from a higher probability of creation of the charged exciton states for higher hole densities. However, the description of the mechanism which 
causes the accompanying attenuation of the neutral exciton line is not obvious. To shed more light on this problem, we chose to change the occupation of the excitonic states; this was realized in pump-probe experiments.

\section{Pump-probe experiments}

We start our discussion with the case where the pump pulse is tuned to resonance with the charged exciton line. This means that the occupation of the charged exciton state is directly modified by the pump pulse. As the temperature is low with respect to $X^{+}-X$ dissociation energy, no neutral exciton population is created during the evolution of the system after excitation. Example results obtained in this experimental configuration are presented in Fig. 3. We show the
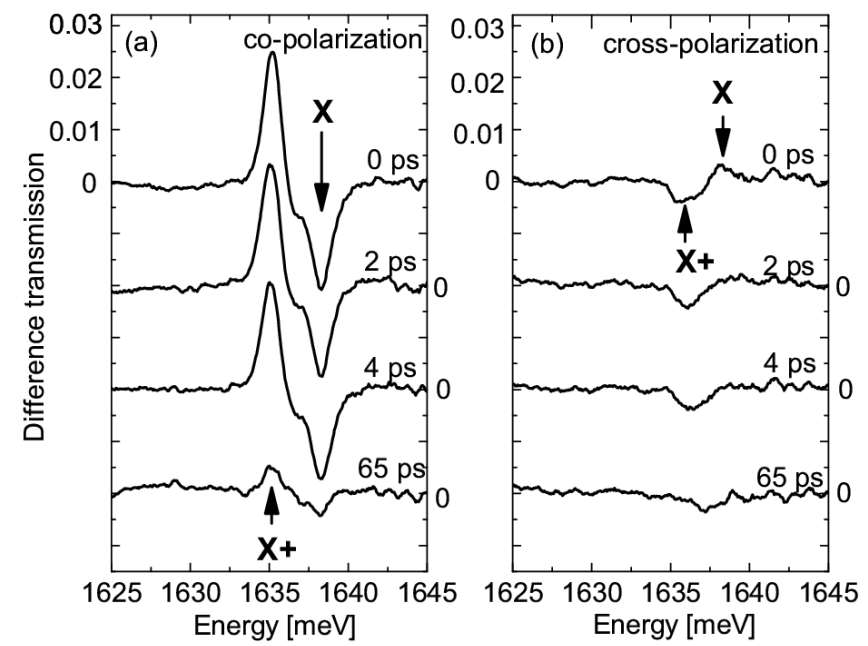

Fig. 3. Pump-probe difference transmission spectra for co-polarized (a) and cross-polarized (b) beams. The pump pulse was tuned to the charged exciton energy.

difference transmission spectra for four different delays between the pump and probe pulses ( $0 \mathrm{ps}, 2 \mathrm{ps}, 4 \mathrm{ps}$ and $65 \mathrm{ps})$. The difference spectra were obtained in this case by subtracting the spectrum at a negative delay (before the pump pulse arrival) from the spectra at a given, positive delay. Thus we can easily follow the temporal evolution of the absorption lines. For a quantitative analysis, both the energy and the intensity of the lines were plotted.

Spectra presented in Fig. 3 were obtained for a hole density $p=3 \times$ $10^{10} \mathrm{~cm}^{-2}$. The intensity of the neutral exciton line increases (negative signal in difference spectra) under strong optical excitation in co-polarization. Simultaneously the intensity of the charged exciton decreases (positive signal in difference spectra). We do not observe such strong effects in cross-polarization. In the latter 
case the intensity of the charged exciton slightly increases, and the intensity of the neutral exciton does not change significantly.

When the pump pulse is tuned to resonance with the charged exciton transition, preexisting holes are directly bound into charged exciton complexes. The density of photocreated excitons is not much less than that of preexisting holes, so that a large number of holes from one spin subband is bound into the charged excitons. Therefore, when the probe pulse has the same polarization as the pump pulse, the transition due to the charged exciton is significantly blocked. Further, we assign the observed increase in the neutral exciton intensity to the binding of free holes into the trions. By changing the occupation of the charged exciton states in such pump-probe experiments, we modify the density of free holes, which has the same effect as reducing the hole density in cw experiments. Therefore, we exclude such effects as phase-space filling, which could be considered as a possible explanation of $\mathrm{cw}$ experiments. We conclude that the neutral exciton absorption is decreased by the interaction of excitons with the free carriers. Without entering into the microscopic nature of many-body interactions, we consider that this mechanism corresponds to the screening of the excitons by free holes [16]. This interpretation can be used if we do not restrict the term of screening to a uniform, spin independent effect, as was done in early studies [20]. Oscillator strength transfer, as a consequence of the Coulomb interaction between carriers, is included in this phenomenological screening.

The results are quite different for cross-polarization. Preexisting holes with spin opposite to that of the photocreated hole screen the exciton more efficiently. In cross-polarization those holes are not bound into the charged exciton by the pump pulse, so that the relevant density of free holes is not changed and the exciton intensity keeps constant. Therefore, screening as described above is spin dependent.

Note that as charged excitons contain two holes with opposite spins (singlet state), they are equally affected by the presence of holes of either spin. Therefore, when holes with any spin are bound into the charged excitons by the pump pulse, screening of the charged exciton is reduced: we observe a small increase in the charged exciton intensity at small positive delays. However, this effect is much weaker than that observed for neutral excitons.

Let us discuss now the resonant excitation of the neutral exciton. The difference transmission spectra, for four different delays, are presented in Fig. 4. Results were obtained for a hole gas density $p=3 \times 10^{10} \mathrm{~cm}^{-2}$. In co-polarization (Fig. 4a), we observe a decrease in the charged exciton intensity. However, the intensity of the neutral exciton does not increase instantaneously, as observed for resonant excitation of the charged exciton. Now, trion formation is a two-step process. In the first step, neutral excitons are created by light. In the second step, preexisting holes are trapped by excitons and trions are formed, as shown schematically in Fig. 5b. When holes are bound into the charged excitons, the 

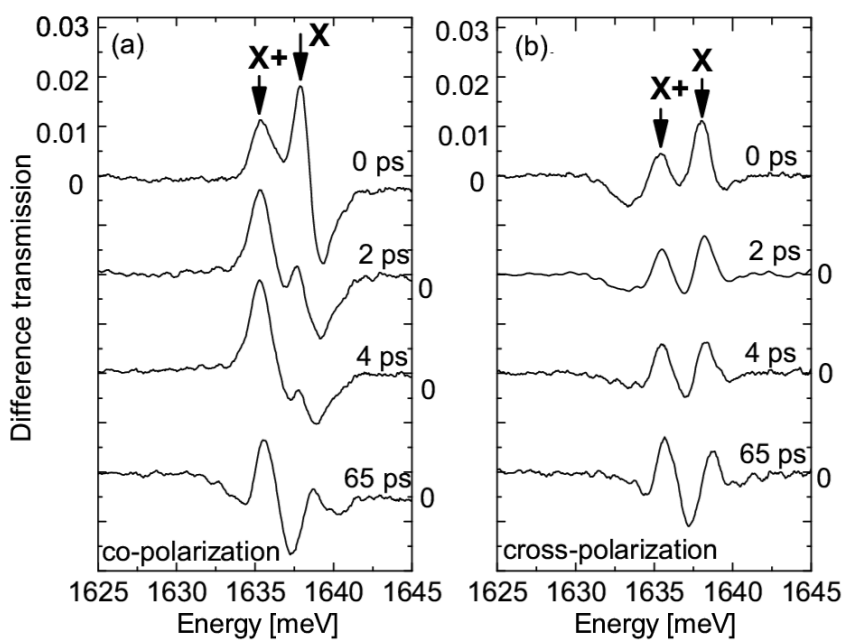

Fig. 4. Pump-probe difference transmission spectra for co-polarized (a) and cross-polarized beams (b). The pump pulse was tuned to the neutral exciton energy; hole gas density $3 \times 10^{10} \mathrm{~cm}^{-2}$.

corresponding screening of the neutral exciton is reduced. Therefore, the rise time of the increase in the neutral exciton intensity coincides with the charged exciton formation time.

The charged exciton formation time depends on the hole density. In Fig. 5a, the total intensity of the neutral exciton is plotted versus time for three different values of the hole density. It is visible that the higher the hole gas density, the

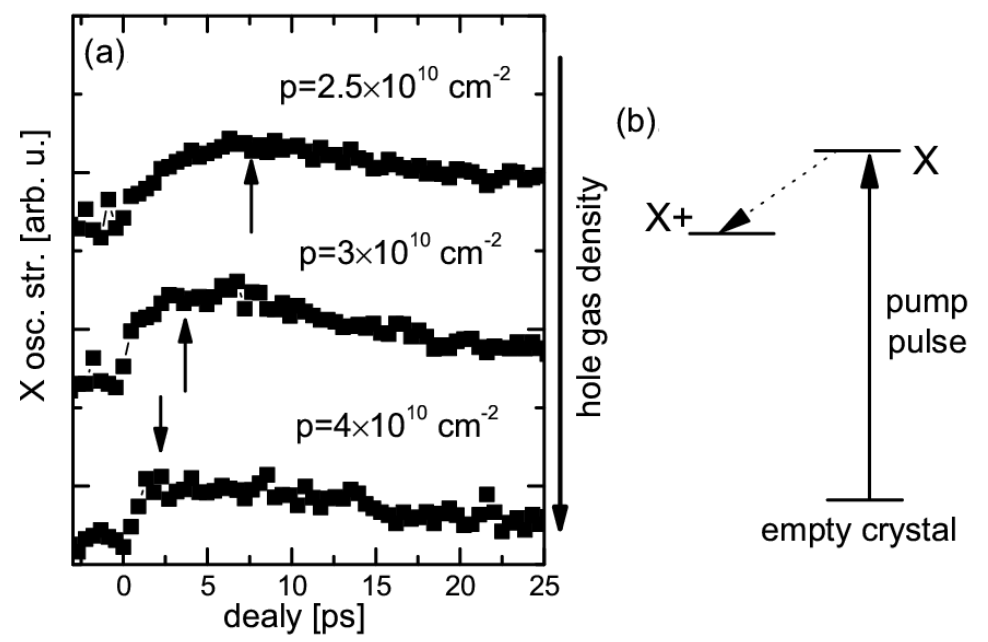

Fig. 5. Oscillator strength of the neutral exciton for different values of the hole density (a). Mechanism of formation of the charged exciton (b). 
faster the charged exciton formation: it is more probable that a neutral exciton traps an additional hole and forms a charged exciton when the density of holes is higher [15].

Effects observed in cross-polarization will not be discussed here. In contrast to the results obtained in the case of resonant excitation of the charged exciton, we observe a decrease in both the neutral and charged exciton intensities (Fig. 4b). The decrease in the trion intensity might be due to screening by photocreated excitons. The decrease in the neutral exciton intensity might be explained by biexciton formation [21].

To sum up the results presented in Figs. 3-5, the influence of the free carriers on the neutral and charged exciton absorption was analyzed. The density of free carriers was changed by binding holes into trions. The intensity of the neutral exciton decreases due to screening by free holes: binding these holes into the charged excitons excludes them from interacting with the rest of the system, which results in an increase in the neutral exciton intensity. By analyzing the different circular polarizations, we confirm that the neutral exciton is more efficiently screened by holes with a spin opposite to that of the hole engaged in the neutral exciton [16].

The effects arising from the interaction with carriers are the strongest ones in such a structure. However, it is also interesting to analyze weaker effects, coming from exciton-exciton interactions. We did it in experiments with resonant excitation of the neutral exciton, performed at hole density values as low as possible $\left(p=0.5 \times 10^{10} \mathrm{~cm}^{-2}\right)$. For the sake of simplicity, we focus on effects at times shorter than the charged exciton formation time. This way we kept the hole

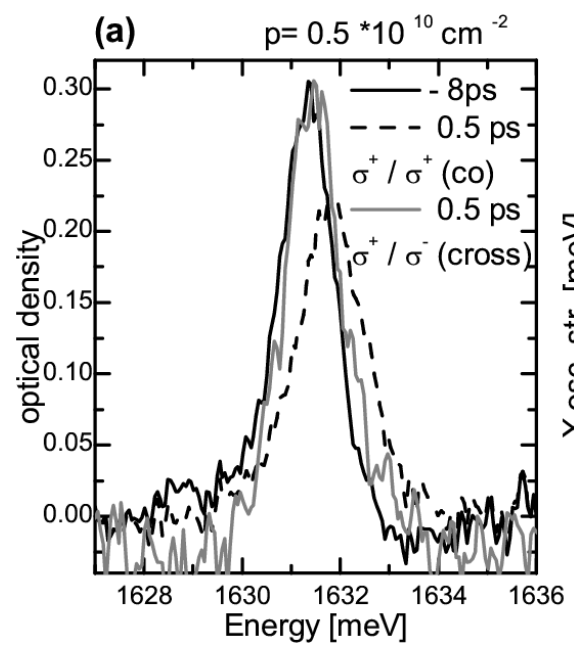

(b)

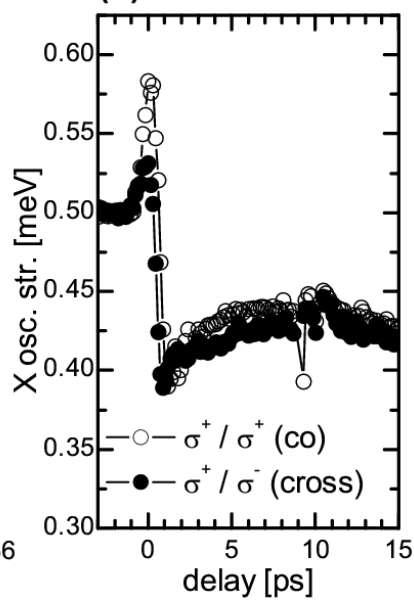

Fig. 6. (a) Optical density for an empty QW for negative delays (black line) and short positive delays (dash line for co-polarization, grey line for cross-polarization). (b) Time evolution of the integrated intensity of the neutral exciton for co-polarized (open circles) and cross-polarized beams (closed circles). 
density constant. Figure 6a shows the optical density of the neutral exciton in negative and small positive delays, for co- and cross-polarizations. The intensity of the neutral exciton decreases in a similar way for both configurations. The evolution of the neutral exciton absorption intensity is presented in Fig. 6b.

In addition, the neutral exciton energy shifts to higher energies. This blue shift is stronger for co- than for cross-polarization.

We ascribe the decrease in the neutral exciton intensity in co-polarized spectra to phase-space filling [20]. Due to the Pauli exclusion principle, excitons cannot be too close to each other (the interaction is repulsive). This results also in the increase in the transition energy.

In cross-polarization the decrease in the neutral exciton line intensity might be due to the biexciton formation [22]. The interaction between excitons with opposite spins is attractive, therefore the energy shift is smaller in this case. However the fact that the change of neutral exciton intensity is the same for both polarization configurations is surprising and calls for deeper theoretical analysis.

\section{Summary}

We have shown that time-resolved absorption measurements are a valuable source of information on exciton-carrier and exciton-exciton interactions in a semiconductor quantum well. This allowed us to propose a microscopic interpretation of oscillator strength stealing effect in terms of spin-dependent screening [16]. Our results show that the carrier induced effects on excitonic optical transitions are more important than the effects of exciton-exciton interaction.

\section{Acknowledgments}

This work has been partially supported by the State Committee for Scientific Research grants PBZ-KBN-044/P03/2001 and 2P03B 002 25, and Polonium program.

\section{References}

[1] K. Kheng, R.T. Cox, Y. Merle d'Aubigné, F. Bassani, K. Saminadayar, S. Tatarenko, Phys. Rev. Lett. 71, 1752 (1993).

[2] G. Finkelstein, H. Shtrikman, I. Bar-Joseph, Phys. Rev. Lett. 74, 976 (1995).

[3] A.J. Shields, J.L. Osborne, M.Y. Simmons, M. Pepper, D.A. Ritchie, Phys. Rev. B 52, 5523 (1995).

[4] A. Haury, A. Arnoult, V.A. Chitta, J. Cibert, Y. Merle d'Aubigné, S. Tatarenko, A. Wasiela, Superlatt. Microstruc. 23, 1097 (1998).

[5] P. Kossacki, J. Cibert, D. Ferrand, Y. Merle d'Aubigné, A. Arnoult, A. Wasiela, S. Tatarenko, J. A. Gaj, Phys. Rev. B 60, 16018 (1999). 
[6] T. Wojtowicz, M. Kutrowski, G. Karczewski, J. Kossut, Appl. Phys. Lett. 73, 1379 (1998).

[7] G.V. Astakhov, D.R. Yakovlev, V.P. Kochereshko, W. Ossau, J. Nürnberger, W. Faschinger, G. Landwehr, Phys. Rev. B 60, R8485 (1999).

[8] R.T. Cox, R.B. Miller, K. Saminadayar, T. Baron, Phys. Rev. B 69, 235303 (2004).

[9] H.P. Wagner, H.P. Tranitz, R. Schuster, Phys. Rev. B 60, 15542 (1999).

[10] P. Gilliot, D. Brinkmann, J. Kudrna, O. Crégut, R. Lévy, A. Arnoult, J. Cibert, S. Tatarenko, Phys. Rev. B 60, 5797 (1999).

[11] S. Adachi, T. Tsuchiya, H. Mino, S. Takeyama, G. Karczewski, T. Wojtowicz, J. Kossut, Physica E 10, 305 (2001).

[12] V. Ciulin, P. Kossacki, S. Haacke, J.-D. Ganière, B. Deveaud, A. Esser, M. Kutrowski, T. Wojtowicz, Phys. Rev. B 62, R16310 (2000).

[13] V. Ciulin, P. Kossacki, M. Kutrowski, J.-D. Ganière, T. Wojtowicz, B. Deveaud, Phys. Status Solidi 229, 627 (2002).

[14] P. Kossacki, H. Boukari, M. Bertolini, D. Ferrand, J. Cibert, S. Tatarenko, J.A. Gaj, B. Deveaud, V.Ciulin, M. Potemski, cond-mat/0404490 (2004).

[15] P. Kossacki, J. Phys. C 15, R471 (2003) and references cited therein.

[16] P. Płochocka, P. Kossacki, W. Maślana, J. Cibert, S. Tatarenko, C. Radzewicz, J.A. Gaj, Phys. Rev. Lett. 92, 177402 (2004).

[17] M.T. Portella-Oberli, V. Ciulin, J.H. Berney, B. Deveaud, M. Kutrowski, T. Wojtowicz, Phys. Rev. B 69, 235311 (2004).

[18] K. Kheng, Ann. Phys. (France) 20 Colloq., 229 (1995).

[19] T. Brunhes, R. André, A. Arnoult, J. Cibert, A. Wasiela, Phys. Rev. B 60, 11568 (1999).

[20] S. Schmitt-Rink, D.S. Chemla, D.A.B. Miller, Phys. Rev. B 32, 6601 (1985).

[21] M. Saba, F. Quochi, C. Ciuti, U. Oesterle, J.L. Staehli, B. Deveaud, G. Bongiovanni, A. Mura, Phys. Rev. Lett. 85, 385 (2000).

[22] P. Kossacki, P. Płochocka, B. Piechal, W. Maślana, A. Golnik, J. Cibert, S. Tatarenko, J.A. Gaj, to be published. 